\title{
A case of unresectable small cell lung cancermore than 7 years of survival treated with cryoablation and low dose chemotherapy
}

\author{
Quanwang Li ${ }^{1}$, Tian Zhou ${ }^{1}$, Min Jiang', Chuanbo Liu ${ }^{1}$, Kaiwen $\mathrm{Hu}^{1 *}$, Franco Lugnani ${ }^{2}$ and Antonio Cueto ${ }^{3}$ \\ ${ }^{1}$ Department of Oncology, Dongfang Hospital, Beijing University of Chinese Medicine, China \\ ${ }^{2}$ International Society of Cryosurgery, Slovenia \\ ${ }^{3}$ Surgery Department Granada University, UGC Thoracic Surgery, Universitary United Hospital of Granada, Spain
}

\begin{abstract}
Small cell lung cancer (SCLC) is common in oncology clinical practice, which is characterized by high malignant level and poor prognosis. Typically, patients with SCLC rarely survive more than a few months without effective treatment. The median survival for patients with limited disease SCLC is only $15-20$ months, with the 5 -year survival rate $10 \%-13 \%$. Here we report a case, female, 58 years, with unresectable limited stage SCLC $(6.5 \mathrm{~cm} \times 5.5 \mathrm{~cm}$, in the right middle lung), who survived from 5 cycles of low doses of chemotherapy, 2 cryosurgery and botanical drugs therapy, living with the primary tumor stably more than 7 years and holding great quality of life.
\end{abstract}

\section{Introduction}

Small cell lung cancer (SCLC) is common in oncology clinical practice, which is characterized by high malignant level and poor prognosis [1]. Typically, patients with SCLC rarely survive more than a few months if without effective treatment. The median survival for patients with limited disease SCLC is only 15-20 months, with the 5-year survival rate 10\%-13\% [2]. We report an unresectable limited stage SCLC case, who survives more than 7 years after the primary tumor was found. During that period, the patient endured 5 cycles of low-dose chemotherapy, 2 cryosurgery and botanical drugs therapy. No evidence of distant metastasis has been found.

\section{Presentation of case}

The patient is a 58 year-old female, weighted $58 \mathrm{~kg}$ and $1.55 \mathrm{~m}^{2}$ in body surface area. Because of cerebral infarction, she was admitted to hospital on Jan. 28,2008 . A $6.5 \mathrm{~cm} \times 5.5 \mathrm{~cm}$ mass was noted in the right middle lung from the computed tomography (CT) scanning on Jan 29, 2008. Though the serum neuron-specific enolase (NSE) level was in normal range, we suspected that the tumor could be malignant. A percutaneous lung biopsy was subsequently performed and the pathological diagnosis of biopsy tissues confirmed that it wasSCLC. No distant metastasis was identified. No abnormalities were noted in the complete blood cell (CBC) count with differential, the serum electrolyte, the renal function and liver function tests. According to the Veterans Administration Lung Group (VALSG) staging system, this patient was diagnosed with limited-stage SCLC $[3,4]$.

\section{Treatment and evaluation}

In contrast to the extensive-stage disease, SCLC patients in limitedstage may benefit more from aggressive, potentially curative treatments $[5,6]$. The management typically involves combination platinum-based chemotherapy and thoracic radiation therapy given with curative intent
$[7,8]$. For this special case, radical surgery was not performed because of the primary tumor was contiguous to the pericardium (Figure 1). Chemotherapy was more proper for her situation. Carboplatin (CBP)/ etoposide(VP-16) regimen (EP) is considered as one of the standard platinum-based combinations for SCLC. However, the patient's performance status (PS) score was only 2, not suitable for the standard doses. We decided to use EP regimen with adjusted doses instead. The first two cycles of chemotherapy were started from Feb. 18, 2008 (CBP

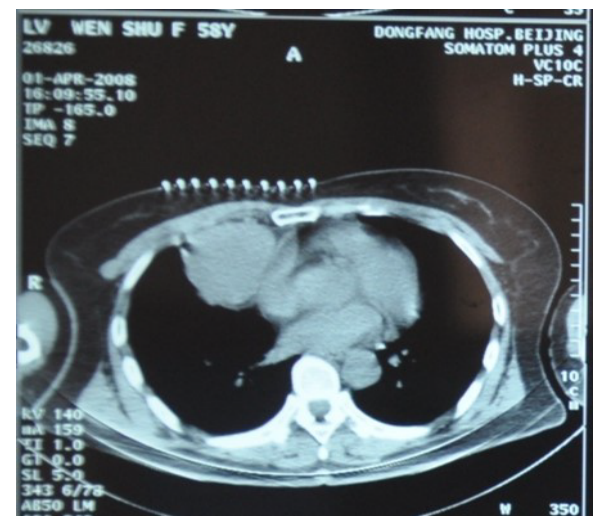

Figure 1. Pre-cryoablation (Apr 1, 2008).

Correspondence to: Kaiwen $\mathrm{Hu}$, Department of Oncology, Dongfang Hospital, Beijing University of Chinese Medicine, 6 Fangxingyuan, No.1 Community, Fengtai District, Beijing 100078, China, E-mail: drkaiwenhu@126.com

Key words: small cell lung cancer, cryoablation, cryosurgery, low dose chemotherapy, comprehensive management, green therapy, long survival

Received: August 20, 2015; Accepted: September 15, 2015; Published: September 19, 2015 
300 mg on Day 1; VP-16 100 mg on Day 1-Day 5; 21 days/cycle; 2 cycles in total).

The CT examination taken after the second cycle of chemotherapy, showed that the tumor size reduction was less than $30 \%$, and the therapeutic evaluation was just stable (SD), much lower than our expectation. During the two cycles of chemotherapy, the patient encountered moderate gastrointestinal adverse reaction, and the WBC count was dropped to $2.8 \times 10^{9} / \mathrm{L}$. After the two cycles, the patient had to spend more than half of her daytime in bed and the PS score was dropped to 3.

In consideration of the unsatisfied efficacy of combination chemotherapy and the induced side effect, we suspend the planned EP regimen and switched to percutaneous cryosurgery, a minimally invasive treatment, to control the primary tumor. In consideration of the primary tumor was contiguous to the pericardium, multiple-partialablation plan was designed after department discussion. The patient received the first cryosurgery on Apr 1, 2008 (Figure 2), and recovered one week later (PS score $=2$ ). Since the patient felt "much better", we tried to give her more cytotoxic drugs. The third cycle of chemotherapy was started from Apr. 8, with the same regimen and doses as the first two. After the fourth cycle of chemotherapy was started from Apr. 29, her PS score was raised to 1 again. We then adjusted the dose of CBP up to $400 \mathrm{mg}$ on Day 1. The CT scan on May 6 showed large area of low-density region in the primary lesion; no significant changes with volume of the tumor. The enhanced perimeter suggested residual tumor existed. And the fifth (last) cycle of chemotherapy was started from May 19, 2008, with the same doses as the fourth cycle.

The second partial-cryosurgery was performed on May 29, 2008. The CT scans on Jul. 5, 2008 and Aug. 28, 2008, demonstrated that, there were no significant changes of the tumor volume; large area of low-density necrosis was noted in the tumor; there were residual tumors near the diaphragm and the cardiac margin. In consideration of that the patient encountered the second degree of myelosuppression and five cycles of EP regimen were performed with unsatisfied efficacy, we decided to stop chemotherapy and observed closely instead. If there were any evidence to show tumor progression, we would consider other chemotherapy regimens. Thereafter, reexaminations of CT scan and NSE level every half-year. Calcification foci have been noted in the center of cryo-zone since Jun 2010, sized $5 \mathrm{~cm} \times 4 \mathrm{~cm}$, while no other remarkable changes (Figure 3-7).

\section{Discussion}

Local treatment approaches such as surgery are the first

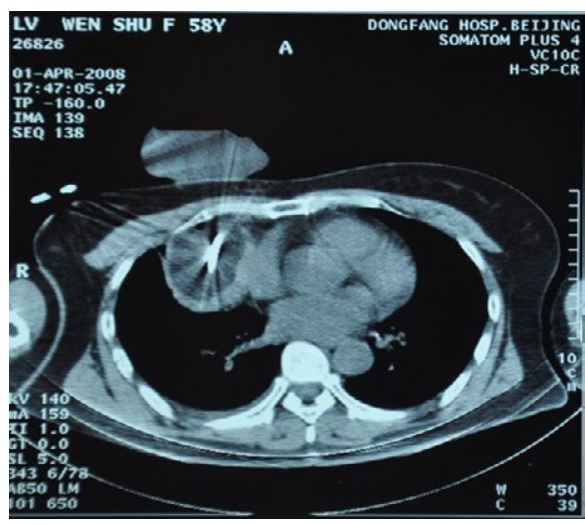

Figure 2. Cryoablation (Apr 1, 2008).

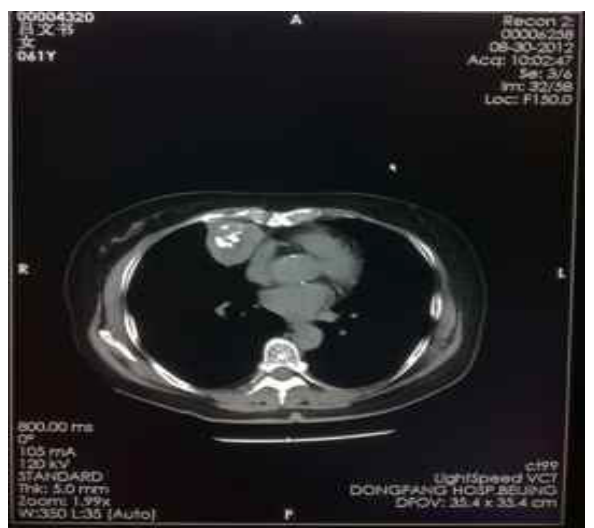

Figure 3. Aug-30-2012.

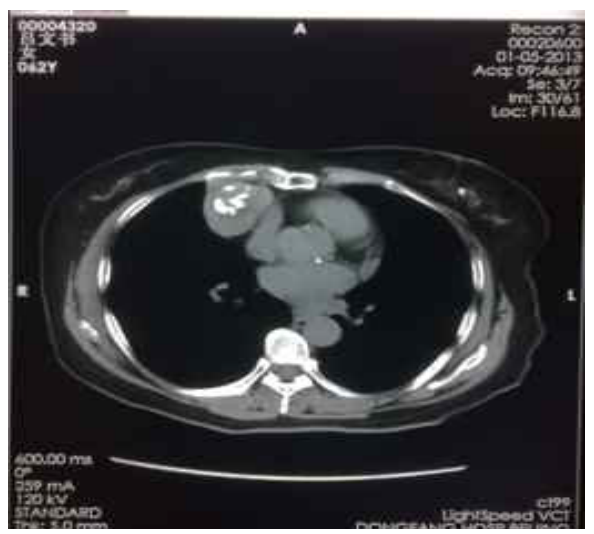

Figure 4. Jan-05-2013.

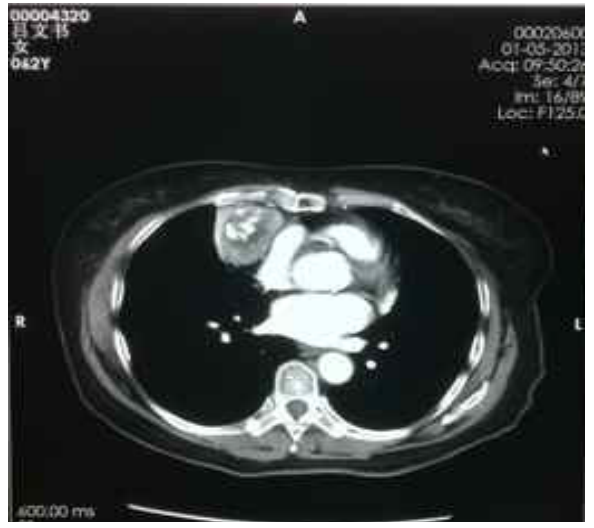

Figure 5. Jan-05-2013 contrast-enhanced.

considerations for patients with limited-stage SCLC. But in this case, a tumor close to the pericardiac plus low performance status lowered the chance to achieve a complete resection. So we turned to expect the potential benefit from combination chemotherapy. SCLC is perceived to be highly responsive to platinum-based combination chemotherapy, and the response rate is higher than $80 \%$. Generally, more than half of limited-stage SCLC patients could get complete response after the chemotherapy. This patient, however, was far away from this goal after several cycles. Though we adjusted the doses according to her situation, she still found it was too hard to tolerate the side effect and wanted to give up the chemo. The primary tumor was not response effectively to the planned regimen as we expected; meanwhile, the combination chemotherapy significantly reduced her quality of life. Typically 


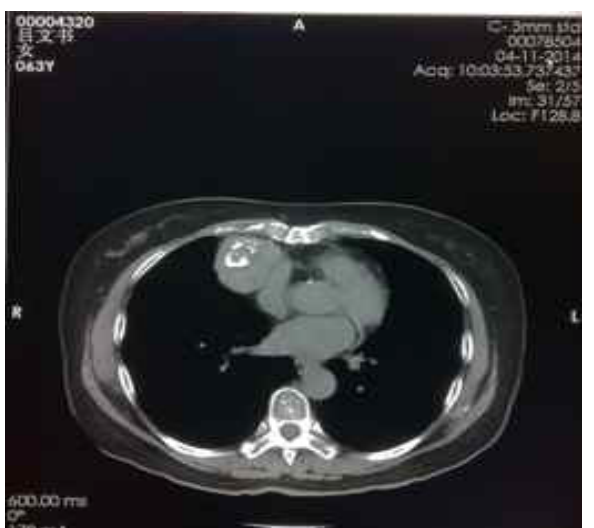

Figure 6. Apr-11-2014.

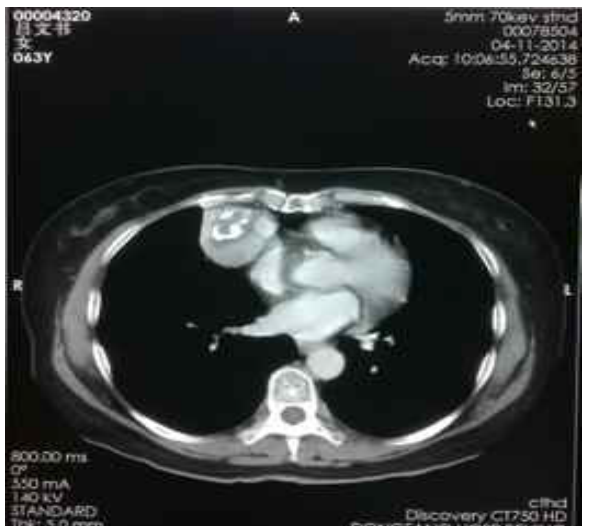

Figure 7. Apr-11-2014 contrast-enhanced.

in such kind of cases, patients will refuse to continue the planned chemotherapy, and what we can offer is the best supportive care (BSC), in a conventional way.

What else can we offer besides the BSC? Is there any other approach to control the incompliant primary tumor more effectively without adding additional pain to the patient? Always, it is the question. In department case discussion held specially for this case after her second cycle of chemo, a consensus was reached: drop the ambitions of complete resection; lower our expectation of combination chemo to SD; let "tenderer" /less-aggressive approaches play the protagonist, instead of chemo; and more, focus on patient's quality of life. Relative to short-term efficacy, we were more willing to pursue patients' longterm survival with tumor. Therefore, the percutaneous multiplepartial-ablation plan was chosen.

The considerations during the patient's management and possible mechanisms were as follows. For the cryoablation, only one part of the tumor was frozen at a time in case of unexpected injury. Rather than to cure the cancer, the purpose of our approach was to alleviate the tumor burden effectively with minimized risks and injuries. During the intervals, we use low-dosed EP regimen to obtain disease stabilization, because of some systematic reviews suggest that administration of cytotoxic drugs at low doses, which has a low toxicity profile, could be an option for some cancer patients [9]. This approach affects the endothelium of the growing tumor vasculature and stimulates the anticancer immune response. Both the antiangiogenic and the immunomodulatory effectcould induce to tumor dormancy, which may improve the patient outcome [10].
Tumor dormancy phenomenon has been observed in many malignancies [11]. The key is that not only a few cells staying in $\mathrm{G}_{0}$ stage [12], but more important, the dynamic balance between proliferation and apoptosis in the tumor cell colonies [13].

It is well known that the progress of cancer is strongly linked to tumor-induced angiogenesis $[14,15]$. Typically, the lowest temperature in a cryoablation process is below $-145^{\circ} \mathrm{C}$, which could cut the blood supply of tumor cells in a couple of minutes, damaging the tumor vascular seriously, and inducing angiogenesis tumor dormancy [16]. Meanwhile, tumor cells would release antigens, which could stimulate the immune system anti-cancer effect via activating CD8+ $\mathrm{T}$ cells, tumor related antigen specific cytotoxic $\mathrm{T}$ cells and etc. $[17,18]$. The Immunological surveillance induced can identify specific enemy cells, control metastasis happens, and induce individual tumor cell dormancy [19].

The concept of conventional chemotherapy is about cytotoxic effects with full doses if one can tolerate. While killing the tumor cells, it is also suppress the patients' immune function. Low-dose chemo, instead, could suppress those immunosuppressed cells, such as suppressor $\mathrm{T}$ cells (Ts), regulatory $\mathrm{T}$ cell (Treg), etc. and down regulate the expression of vascular endothelial growth factor (VEGF), and reduce the microvascular density [20]. All these mechanisms favor the stability of cancer.

This strategy was effective in this unresectable SCLC case. It has been more than 7 years since the patient was diagnosed with SCLC, and she has kept great quality of life ever after the treatment (PS score $=0$ ).

This suggest that if the tumor dormancy could be kept for a long while, the goal of long-term survival with tumor could be achieved, partially at least. In the other word, if we want to make the dream of long-term survival with tumor to be realistic, it was not necessary to kill all the cancer cells or focus too much on the volume reduction of the mass. It was more feasible to induce tumor dormancy and keep this status as long as possible. We shall remember that, it is the only lighthouse that directs our works is the long-term survival with fair quality of life of our patients.

\section{Acknowledgements}

We would like to express our gratitude to Dr. Franco Lugnani and Dr. A. Cueto for their inspiration, discussion of study and revision of this manuscript.

\section{References}

1. Cueto A, Burigana F, Nicolini A, Lugnani F1 (2014) Neuroendocrine tumors of the lung: hystological classification, diagnosis, traditional and new therapeutic approaches. Curr Med Chem 21: 1107-1116. [Crossref]

2. Lally BE, Urbanic JJ, Blackstock AW, Miller AA, Perry MC (2007) Small cell lung cancer: have we made any progress over the last 25 years? Oncologist 12: 1096-1104. [Crossref]

3. Micke P, Faldum A, Metz T, Beeh KM, Bittinger F, et al. (2002) Staging small cell lung cancer: Veterans Administration Lung Study Group versus International Association for the Study of Lung Cancer--what limits limited disease? Lung Cancer 37: 271-276. [Crossref]

4. American Cancer Society. Lung cancer (small cell): how is small cell lung cance staged? Available at http://www.cancer.org/cancer/lungcancer-smallcell/detailedguide/ small-cell-lung-cancer-staging. Accessed on Aug 24, 2014.

5. Pillai RN, Owonikoko TK (2014) Small cell lung cancer: therapies and targets. Semin Oncol 41: 133-142. [Crossref]

6. Simos D, Sajjady G, Sergi M, Liew MS, Califano R, et al. (2014) Third-line chemotherapy in small-cell lung cancer: an international analysis. Clin Lung Cancer 15: 110-118. [Crossref] 
7. Kalemkerian GP (2014) Advances in pharmacotherapy of small cell lung cancer. Expert Opin Pharmacother 15: 2385-2396. [Crossref]

8. Rossi A, Martelli O, DiMaio M (2013) Treatment of patients with small-cell lung cancer: from meta-analyses to clinical practice. Cancer Treat Rev 39: 498-506. [Crossref]

9. Montagna E, Cancello G, Dellapasqua S, Munzone E, Colleoni M (2014) Metronomic therapy and breast cancer: a systematic review. Cancer Treat Rev 40: 942-950. [Crossref]

10. Berruti A, Sperone P, Bellini E, Daffara F, Perotti P, et al. (2011) Metronomic therapy concepts in the management of adrenocortical carcinoma. Horm Cancer 2: 378-384. [Crossref]

11. Naumov GN, MacDonald IC, Chambers AF, Groom AC (2001) Solitary cancer cells as a possible source of tumour dormancy? Semin Cancer Biol 11: 271-276. [Crossref]

12. Murray C (1995) Tumour dormancy: not so sleepy after all. Nat Med 1: 117-118 [Crossref]

13. Holmgren L, O'Reilly MS, Folkman J (1995) Dormancy of micrometastases: balanced proliferation and apoptosis in the presence of angiogenesis suppression. Nat Med 1: 149-153. [Crossref]
14. McDougall SR, Anderson AR, Chaplain MA (2006) Mathematical modelling of dynamic adaptive tumour-induced angiogenesis: clinical implications and therapeutic targeting strategies. $J$ Theor Biol 241: 564-589. [Crossref]

15. Pencovich N, Hantisteanu S, Wurtzel O (2013) Unique expression patterns associated with preferential recruitment of immature myeloid cells into angiogenic versus dormant tumors. Genes Immun 14: 90-98

16. Bagri A, Berry L, Gunter B, Singh M, Kasman I, et al. (2010) Effects of anti-VEGF treatment duration on tumor growth, tumor regrowth, and treatment efficacy. Clin Cancer Res 16: 3887-3900. [Crossref]

17. Finn OJ (2006) Human tumor antigens, immunosurveillance, and cancer vaccines. Immunol Res 36: 73-82. [Crossref]

18. Farrar JD, Katz KH, Windsor J, Thrush G, Scheuermann RH, et al. (1999) Cance dormancy. VII. A regulatory role for CD8+ T cells and IFN-gamma in establishing and maintaining the tumor-dormant state. J Immunol 162: 2842-2849. [Crossref]

19. Röcken M (2010) Early tumor dissemination, but late metastasis: insights into tumo dormancy. J Clin Invest 120: 1800-1803. [Crossref]

20. Cerullo V, Diaconu I, Kangasniemi L, Rajecki M, Escutenaire S, et al. (2011) Immunological effects of low-dose cyclophosphamide in cancer patients treated with oncolytic adenovirus. Mol Ther 19: 1737-1746. [Crossref]

Copyright: (C) $2015 \mathrm{Li} \mathrm{Q}$. This is an open-access article distributed under the terms of the Creative Commons Attribution License, which permits unrestricted use, distribution, and reproduction in any medium, provided the original author and source are credited. 